\title{
Studien über den Gehalt verschiedener Pilzpreßsäfte an Oxydasen.
}

\author{
Von
}

Hans Pringsheim.

(Aus dem chemischen Institut der Universität Berlin.)

(Der Redaktion zugegangen am 12. August 1909.)

Die in vorstehender Abhandlung beschriebene Darstellung von Pilzpreßsäften, welche noch durch einige von anderen Pilzen ergänzt wurde, gab mir Gelegenheit, den Gehalt dieser Säfte an Oxydasen zu prüfen. Ich beschränkte mich dabei auf den Nachweis der Katalase, Oxydase, Peroxydase und Tyrosinase. Die Anwesenheit der Katalase wurde ermittelt, indem einige Tropfen der Pilzpreßsäfte zu $2-3 \mathrm{ccm}$ Wasserstoffsuperoxyd gegeben wurden, wobei sofort oder nach einiger Zeit mehr oder weniger starke Sauerstoffabgabe unter Aufschäumen der Flüssigkeit zu beobachten war. Auf Oxydase wurde mit $2-3 \mathrm{ccm}$ einer etwa $2 \%$ igen frischbereiteten Pyrogallollösung geprüft, der zum Nachweis der Peroxydase die gleiche Menge Wasserstoffsuperoxydlösung zugegeben wurde. Die Anwesenheit der Katalase hebt den Nachweis der Peroxydase nicht auf $;^{1}$ ) eine Verfärbung der Flüssigkeit war auch schon lange zu beobachten, ehe alles Wasserstoffsuperoxyd zerstört sein konnte. Beim Vermischen mit einigen Tropfen der Pilzpreßsäfte trat momentan oder nach einigen Minuten mehr oder weniger starke Färbung auf, deren Intensität durch die in nachfolgender Tabelle markierten Kreuze ausgedrückt wird. Auf diese Weise war Peroxydase nur beim Ausbleiben der Oxydasereaktion mit Sicherheit nachzuweisen. In einigen Fällen deutete aber die Intensität der Färbung, die bei Anwesenheit von Peroxydase mit wenigen Ausnahmen größer

1) R. Ghodat, Arch. science et nat. Genève (4), Bd. XXIII, S. 265. 
war, auf die Anwesenheit von Peroxydase neben Oxydase hin. Mehrfach war auch ein deutlicher Unterschied in der Farbennuance bei Verwendung von Pyrogallol allein oder Pyrogallol plus Wasserstoffsuperoxyd zu bemerken, was gleichfalls in der Tabelle angegeben wird. Auf Tyrosinase wurde mit verdünnter Lösung von l-Tyrosin geprüft; doch konnte in keinem der untersuchten Fälle eine Verfärbung dieser Lösung nachgewiesen werden, auch nicht mit dem Preßsaft von Monilia sitophila, für welchen Pilz Went ${ }^{1}$ ) Tyrosinase nachgewiesen $\mathrm{zu}$ haben scheint.

Wie aus der Zusammenstellung hervorgeht, wurde bei den 17 in den Kreis der Untersuchung gezogenen Pilzen die Katalase $13 \mathrm{mal}$, die Oxydase $9 \mathrm{mal}$ und die Peroxydase in Abwesenheit der 0xydase noch 7 mal nachyewiesen. Weder Oxydase noch Peroxydase wurde nur einmal bei Allescheria Gayonii gefunden.

Trotzdem die Versuchsbedingungen die gleichen waren, gaben die Preßsäfte aus zu verschiedenen Zeiten gezüchteten Pilzen in einigen Fällen nicht dieselben Resultate. So konnte z. B. in den Preßsäften aus Mucor mucedo 2 mal Katalase und 1 mal keine Katalase und 2 mal Oxydase und 1 mal keine Oxydase nachgewiesen werden, während nur einer der drei Preßsäfte aus Penicillium purpurogenum Peroxydasefärbung hervorrief. Der Nachweis dieser Fermente steht daher noch auf schwachen Füßen; nur die positiven Resultate sind zuverlässig. Ich will daher aus den Resultaten fürs erste keinerlei Schlüsse auf die Bedeutung der Oxydasen für die Physiologie der Pilze ziẻhen, was bei dem Stande unserer Kenntnis in bezug auf diesen Punkt nur eine Vermehrung der verwirrenden Theorien ${ }^{2}$ ) über

1) F. C. Went, Über den Einfluß der Ernährung auf die Enzymbildung der Monilia sitophila. Jahrbücher für wissenschaftl. Botanik, Bd. XXXVI (1901), S. 611.

2) Vgl. unter anderem: J. Behrens, Oxydasewirkungen in Lafar, Handbuch der technischen Mykologie, G. Fischer, Jena 1905/1909, Bd. I, S. 668, und die neueste Zusammenstellung über Oxydasen von Gasimir Funk. Neue Ergebnisse auf dem Gebiete der Oxydasen. Medizinische Klinik, Jg. 1909, Nr. 4. Carl Oppenheimer, Die Fermente und ihre Wirkungen. Dritte Aufl., F. C. W. Vogel, Leipzig 1909, S. 337. Die Oxydasen. 


\begin{tabular}{|c|c|c|c|c|c|}
\hline Pilzspezies & $\begin{array}{c}\text { Preß- } \\
\text { saft }{ }^{1} \\
\mathrm{Nr} .\end{array}$ & Katalase & Oxydase & Peroxydase & $\begin{array}{c}\text { Tyrosi- } \\
\text { nase }\end{array}$ \\
\hline $\begin{array}{c}\text { I. Allescheria } \\
\text { Gayonii }\end{array}$ & $\begin{array}{l}1 \\
2 \\
\end{array}$ & $\begin{array}{l}++ \\
++\end{array}$ & - & - & - \\
\hline II. Aspergillus Wentii & $\begin{array}{l}3 \\
4 \\
\end{array}$ & $\begin{array}{l}+++ \\
+++\end{array}$ & - & $+\bar{t}$ & - \\
\hline $\begin{array}{l}\text { III. Rhizopus } \\
\text { tonkinensis }\end{array}$ & $\begin{array}{l}5 \\
5 \mathrm{a}\end{array}$ & - & $\begin{array}{l}\text { schwach } \\
\text { schwach }\end{array}$ & $\begin{array}{l}+++ \\
\text { momentan } \\
+++\end{array}$ & 一 \\
\hline IV. Mucor mucedo & $\begin{array}{l}6 \\
7 \\
8\end{array}$ & $\begin{array}{l}++ \\
- \\
+\end{array}$ & $\begin{array}{c}++ \text { bläulicher } \\
\text { als Peroxydase } \\
- \\
+ \text { bläulicher } \\
\text { als Peroxydase }\end{array}$ & $\begin{array}{l}++ \\
++ \\
+\end{array}$ & \\
\hline V. Mucor corymbifer & 9 & - & - & + & - \\
\hline $\begin{array}{l}\text { VI. Mucor rhizopodi- } \\
\text { formis }\end{array}$ & $\begin{array}{l}10 \\
11 \\
\end{array}$ & schwach & - & $\begin{array}{c}t+t \\
+\end{array}$ & 一 \\
\hline VII. Mucor racemosus & 12 & schwach & sehr schwach & $\underset{\text { momentan }}{+++}$ & - \\
\hline $\begin{array}{c}\text { VIII. Hypomyces } \\
\text { rosellus }\end{array}$ & 13 & + & $+t+$ & + & \\
\hline $\begin{array}{c}\text { IX. Penicillium } \\
\text { africanum }\end{array}$ & 14 & - & sehr schwach & + & - \\
\hline $\begin{array}{c}\text { X. Penicillium } \\
\text { brevicaule }\end{array}$ & 15 & $+t+$ & - & + & 一 \\
\hline XI. Mucor javanicus & 16 & - & $+t+$ & + & \\
\hline $\begin{array}{c}\text { XII. Aspergillus } \\
\text { Oryzae }\end{array}$ & $\begin{array}{l}18 \\
19\end{array}$ & - & - & $\begin{array}{c}+++ \\
\text { dunkelrot- } \\
\text { braun } \\
+++\end{array}$ & \\
\hline $\begin{array}{l}\text { XIII. Penicillium } \\
\text { purpurogenum }\end{array}$ & $\begin{array}{l}20 \\
21 \\
24\end{array}$ & $\begin{array}{c}+ \\
+++ \\
\text { momentan } \\
+++\end{array}$ & - & $\begin{array}{c}- \\
- \\
+\quad \text { nach } \\
+1 / 2 \text { Stunde } \\
\end{array}$ & - \\
\hline XIV. Monilia sitophila & a & + & ++ braun & ++ violett & 一 \\
\hline $\begin{array}{l}\text { XV. Fusarium } \\
\text { vasinfectum }\end{array}$ & b & ++ & bläulich & $\underset{\text { rotbraun }}{+}$ & \\
\hline $\begin{array}{l}\text { XVI. Fusarium } \\
\text { muschatum }\end{array}$ & c & + & $\underset{\text { bläulich }}{+}$ & $\underset{\text { rotbraun }}{+}$ & \\
\hline $\begin{array}{l}\text { XVII. Sclerotinia } \\
\text { cslerotiorum }\end{array}$ & $d$ & ++ & - & schwach & \\
\hline
\end{tabular}

1) Vgl. die vorstehende Abhandlung von H. Pringsheim und G. Zemplén. 
Über den Gehalt verschiedener Pilzpreßsäfte an Oxydasen. 389

die Funktion der Oxydasen bedeuten würde. Vielleicht eignet sich der eine oder der andere Pilz, um in Zukunft detailliertere Studien über die Oxydasen mit Hilfe der Pilzpreßsäfte anzustellen.

Ich gebe hier noch die Züchtungsbedingungen der in der vorstehenden Abhandlung nicht geprüften Pilze. Der Preßsaft aus Sclerotinia zeichnete sich durch besondere Schwerflüssigkeit und Klebrigkeit vor allen andern Preßsäften aus.

XIV. Monilia sitophila.

Preßsaft a) Nährlösung Pflaumendekokt. Wachstum 26. Mai bis 8 . Juni.

XV. Fusarium vasinfectum.

Preßsaft b) Nährlösung Rohrzucker, Pepton. Wachstum 5. Juli bis 3. August.

XVI. Fusarium muschatum.

Preßsaft c) Nährlösung Rohrzucker, Pepton. Wachstum 15. Juli bis 5. August.

XVII. Sclerotinia sclerotiorum.

Preßsaft d) Nährlösung Rohrzucker, Pepton, schwefelsaures Ammoniak. Wachstum 10. Juli bis 4. August. 